\title{
EL PRINCIPIO DE JUSTICIA Y LA SALUD EN CHILE
}

\author{
Claudio Lavados Montes* y Alejandra Gajardo Ugás*
}

Resumen: La equidad en el acceso a la atención de salud, eficacia en las intervenciones destinadas a promover, preservar y recuperar la salud, y eficiencia en el uso de los recursos disponibles son los objetivos centrales de la Reforma del Sistema de Salud en Chile, basados en el principio de justicia. Este artículo trata el tema de la justicia en salud desde la definición tradicional, pasando por la de salud, para culminar en lo que la bioética entiende hoy por justicia, y si este principio se verifica o es vulnerado dentro del Plan AUGE, toda vez que la Constitución Política de Chile establece el derecho a la salud como un derecho básico reconocido para todos los ciudadanos.

Palabras clave: salud, justicia, Plan AUGE

\section{THE PRINCIPLE OF JUSTICE AND HEALTH IN CHILE}

\begin{abstract}
Equality in access to health care, efficiency in interventions designed to promote, preserve and recuperate health, and the efficient use of available health care resources are the central objectives of the Health Care System Reform in Chile, based on the principle of justice. This article deals with the theme of justice in health, starting from the traditional definition, continuing to that of healthcare, and culminating in what bioethics interprets today as justice, and whether this principle is verified or not under Plan AUGE, as the Political Constitution of Chile establishes the right to health care as a basic right recognized for all citizens.
\end{abstract}

Key words: health, justice, Plan AUGE

\section{O PRINCIPIO DA JUSTIÇA E A SAÚDE NO CHILE}

Resumo: Eqüidade no acesso à atenção de saúde, eficácia nas intervençôes destinadas a promover, preservar e recuperar a saúde e eficiência no uso dos recursos disponíveis, são os objetivos centrais da Reforma do Sistema de Saúde no Chile, baseados no principio da justiça. Este artigo trata o tema da justiça em saúde a partir da definição tradicional, passando pela de saúde, para culminar no que a bioética entende hoje por justiça e se este principio se verifica ou é vulnerado dentro do Plano AUGE, toda vez que a Constituição Política do Chile estabelecer o direito à saúde como um direito básico reconhecido para todos os cidadãos.

Palavras-chave: saúde, justiça, Plano AUGE

* Licenciado en Teología. Profesor Universidad Santo Tomás, Chile Correspondencia: claudiolavados@gmail.com

** Licenciada en Enfermería. Directora Escuela de Enfermería, Universidad Santo Tomás, Chile Correspondencia: agajardo@santotomas.cl 


\section{Introducción}

"En el último siglo la salud ha dejado de ser una cuestión que los individuos gestionan privadamente, para convertirse en problema público y político. Por eso los términos 'sanidad' y 'política', en principios ajenos entre sí, han llegado a unirse indisolublemente en la expresión 'política sanitaria'. Hoy es difícil encontrar algún aspecto de la salud de las personas completamente desligado del inmenso aparato burocrático de la política sanitaria. Muchos consideran excesiva esta injerencia de la política en sanidad, en tanto que para otros resulta todavía insuficiente. Pero todos justifican sus puntos de vista apelando al concepto de justicia distributiva. No puede extrańar, por ello, que uno de los capítulos más vivos y polémicos de la bioética actual sea el de la justicia sanitaria. ¿Cuándo debe considerarse justo o injusto un servicio de salud? ¿Qué recursos es preciso asignarle para cumplir con el deber de justicia? ¿Cómo proceder cuando los recursos disponibles son menores de los teóricamente necesarios? ¿Cómo distribuir justamente los recursos insuficientes? He aquí algunas de las preguntas que se hacen diariamente los políticos, los gestores sanitarios y el público en general" (1).

\section{Justicia: definición y perspectiva bioética}

\section{Intento de definición de justicia}

No ha sido posible responder de manera definitiva a la pregunta ¿qué es la justicia? La palabra se emplea de diversas maneras, todas ellas legítimas. Sin embargo, es posible identificar los principales significados del concepto: en primer lugar, como retribución o castigo $y$, segundo, como equidad $y$, en particular, como distribución equitativa.

El primero es más pertinente en discusiones sobre leyes que sobre salud, ya que se relaciona con el hecho de que las personas que hacen algo incorrecto deben responder por sus actos ante la justicia. Aquí el castigo o la retribución se consideran como algo "justo" porque su objetivo es corregir el error que se cometió.

El segundo sentido es el de equidad. En ocasiones se lo describe como la distribución justa de un bien a todos los individuos, en porciones iguales. ¿Cómo podemos interpretar esto? Existe, en cambio, otra interpretación más amplia y significativa del concepto: "a cada cual según sus derechos", la cual se deriva de la idea de que la equidad es un tipo de igualdad. En este caso, el concepto implica que cada individuo tiene los mismos derechos a recibir atención de salud.

Sin embargo, la igualdad en derechos no concede automáticamente igualdad en retribuciones. En salud esto quiere decir que, a pesar de que todos tienen el mismo derecho a recibir atención, ello no asegura de manera automática una distribución equitativa.

\section{Asignación de recursos}

Con frecuencia escuchamos que se cuenta con recursos limitados, pero la demanda es ilimitada. Por tanto, es poco realista esperar que se puedan cubrir todas las demandas en su totalidad. Si comenzamos desde esta posición, entonces es inevitable que exista cierto grado de injusticia, porque no se cubrirán las necesidades de algunas personas y sí se cubrirán las de otras. Pareciera ser que la salud nunca podrá cubrirse de manera total, a menos que se cuente con recursos ilimitados. De hecho, sería preciso contar con más recursos para la probable demanda y de esta forma asegurar una justicia absoluta.

El principio de justicia comprende el uso racional de recursos disponibles que permita el ejercicio pleno del derecho a la salud, lo que apunta no sólo a los ciudadanos, salvaguardando su dignidad de personas, sino a las obligaciones del Estado de asegurar, responsablemente, el mínimo de los mínimos.

Justiniano caracterizó la justicia como el deseo constante y perenne de entregarle a cada uno lo que le es debido. La dificultad estriba en establecer qué se debe a quién y por qué. El principio de justicia exige una distribución "equitativa" de los recursos en la procura del bien "salud". A lo largo de un par de décadas ha ido discurriendo la bioética sobre el significado y el peso de cada uno de estos principios para lograr una buena práctica asistencial y de la investigación. Temas tan centrales y sensibles para la vida humana en situación de enfermedad, como el consentimiento informado, las directrices anticipadas o la confidencialidad, han ido viendo la luz, pero las cuestiones referentes al principio de justicia son las que reclaman una especial atención, porque se encuentra en una situación poco entendida y comprendida, como también de "alto riesgo". Las acusaciones de ineficiencia y despilfarro de que está siendo objeto el sistema de salud pública reclaman, sin duda, reformas que apuesten por una mayor eficiencia y eficacia en la gestión de los recursos. 
La gestión sanitaria sólo será verdaderamente eficiente si se encuadra dentro de un marco de justicia social que se comprometa a cumplir al menos dos requisitos: cubrir unos mínimos universales de justicia sanitaria a los que no puede renunciar una sociedad que se pretenda justa, y percatarse de que para satisfacerlos no bastan las reformas del sistema sanitario en sentido estricto, sino que es necesario que la sociedad en su conjunto apueste por nuevas formas de vida. Por eso el discurso de la eficiencia, verdaderamente ineludible en salud, debe cobrar todo su sentido en un marco de justicia que reclama la satisfacción de unos mínimos irrenunciables y la transformación de la vida social. En efecto, recordemos cómo fue el nacimiento de los estados de bienestar, el que trajo de la mano la convicción de que la asistencia sanitaria es un asunto de justicia social, un asunto público y de prioridad del Estado y no una cuestión privada. Razones diversas abonaron una convicción semejante, desde las puramente economicistas hasta el reconocimiento de dos hechos morales: la salud es un bien tan básico que la atención sanitaria no puede quedar al arbitrio del mercado y, a mayor abundamiento, su financiamiento privado aumenta las desigualdades. La atención sanitaria se reconoce como un derecho humano al menos desde 1948, y el estado social de derecho se juega su legitimidad, entre otras cosas, en procurar atención salud a todos sus miembros.

\section{Teoría de la justicia aplicada en bioética}

John Rawls ("A Theory of Justice", 1971) construye dos principios sobre los cuales basar la noción de justicia social. A partir de una "posición original", equivalente al contrato social de los filósofos políticos clásicos, sostiene que, en términos generales, la idea básica de la justicia es la ausencia de desigualdades arbitrarias. ¿Cuáles serían éstas en el campo de la salud colectiva? Un modo efectivo de distinguirlas podría ser detectarlas en función del desarrollo de cada sociedad, en la distribución de recursos y en el acceso a la salud -en el amplio espectro comprendido en la definición de la Organización Mundial de la Salud.

De las variables utilizadas por Rawls para definir la justicia, una está referida al ámbito del derecho privado, incluyendo la temática de las libertades. Para ello aplica el principio según el cual "todas las personas tienen el mismo derecho a la libertad básica más amplia que sea compatible con la libertad similar de los demás" (se refiere a las libertades de expresión, de asociación, la libertad de conciencia y de pensamiento, derecho a elegir y ser elegido, derecho a la propiedad privada, a no padecer embargo o detención arbitraria, derecho de asociación). La segunda abarca las desigualdades sociales y económicas, base de la estratificación social tanto para teorías sociológicas monistas como pluralistas, e incluye dos principios: a) el de diferencia, según el cual esas diferencias y desigualdades sociales estarán organizadas de modo tal que puedan suponerse, con criterio de realidad, que redundarán en beneficio de todos, y b) el de igualdad de oportunidades, que supone que, ajustadas armoniosamente, dichas diferencias se correspondan con posiciones y cargos accesibles a todos.

\section{Justicia sanitaria}

En materia de salud, ha sido Norman Daniels quien ha intentado aplicar la teoría rawlsiana de la justicia. En su opinión, la igualdad de oportunidades tiene otro bien primario de carácter subsidiario que es el derecho a la asistencia sanitaria. En tal caso podría hablarse de derecho a la justicia sanitaria, entendiendo por tal una "asistencia médica justa" que a su vez requiere la definición clara y precisa de "necesidades de asistencia". Daniels las define desde el "funcionamiento típico de la especie", que serían "todas aquellas desviaciones de la organización funcional natural de un miembro de la especie", pero no todas.

Otro bioeticista que retoma la idea de salud como bien social primario es Ronald Green, quien no sólo la limita a la generación actual sino que la vincula con el deber de preservación de la calidad de vida de las generaciones futuras, a tono con el auge de las teorías medioambientalistas.

En este orden de ideas, Diego Gracia cree que este enfoque puede ser la base fundamental de discusión para la toma de decisiones en materia de justicia sanitaria en el futuro.

\section{Salud en Chile, ¿principio de justicia?}

La Constitución de la República de Chile declara que la salud es un derecho básico reconocido para todos los ciudadanos. En las propuestas de reforma de la atención de salud que se han gestado en el país se la considera como un bien social. La igualdad de oportunidades en la atención de salud es la expresión más clara de cómo se manifiestan y reclaman los derechos de las personas. Por eso se considera que la equidad en salud, así como 
el acceso, calidad, oportunidad y protección financiera, debe constituir un derecho esencial de todas las personas, y un imperativo ético en las políticas de salud.

En lo que se refiere a indicadores de salud, el progreso que ha presentado Chile en las últimas décadas, respecto de la mortalidad infantil, materna y general, es notable, llegando a cifras comparables con las de países desarrollados. Estos importantes logros no han beneficiado de la misma forma a toda la población, existiendo en la actualidad una gran variabilidad de estos indicadores al desagregarlos en los distintos niveles de organización geopolítica, la cual representa la desigualdad del estado de salud de la población chilena(2).

El estudio de las inequidades en salud se ha planteado tanto en el ámbito económico como sanitario. Autores nacionales expresan que la equidad en el nivel de salud de la población representa el indicador central del impacto de la intervención social, el cual tiene un profundo significado ético, filosófico, económico y de justicia social. Margaret Whitehead (OMS) define funcionalmente(3): "Por inequidad en salud se entienden las diferencias sistemáticas que son innecesarias y evitables, además de injustas”.

Inequidad se define como justicia natural en oposición a la letra de la ley positiva. El concepto de inequidad se refiere a la insuficiencia de justicia distributiva -en el concepto aristotélico de igualdad entre iguales- y a la desigualdad de oportunidades de acceso proporcional a determinados servicios o prestaciones -en el concepto de Rawls. Esta última teoría de la justicia considera además que las inevitables desigualdades sociales y económicas no deben recaer en los más desfavorecidos.

Equidad en salud implica el ideal que cada persona tenga la misma oportunidad de obtener su máximo potencial en salud y, más pragmáticamente, que nadie esté en desventaja para alcanzar este potencial si esto puede ser evitable.

La expresión activa de la equidad significa promover la disminución de las brechas en los indicadores de salud que diferencian a los grupos sociales mediante la acción sobre determinantes sociales, económicos, tecnológicos y culturales que afectan a los grupos más desfavorecidos. La estrategia es identificar las brechas de equidad en salud y analizar sus causas, y para ello debemos tener presente que equidad implica crear igualdad de oportunidades en salud y llevar las diferencias en salud al nivel más bajo posible.
La equidad en el acceso a la atención de salud, eficacia en las intervenciones destinadas a promover, preservar y recuperar la salud, y eficiencia en el uso de los recursos disponibles son los objetivos centrales de la reforma de nuestro actual Sistema de Salud.

Dentro de la reforma de salud se encuentra el Plan Auge que plantea como objetivo central garantizar el acceso a atención médica, dentro de plazos estrictos predefinidos(4), a todos los pacientes que cumplan con determinados criterios de inclusión, consistentes en la confirmación diagnóstica y tratamientos estandarizados para un conjunto de enfermedades priorizadas por su alto impacto sanitario y social. Para las prestaciones asociadas se definen, además, garantías explícitas y exigibles de acceso, oportunidad, calidad y cobertura financiera(5).

El desafío radica en que el sistema público de salud pueda cumplir con los plazos establecidos por el Plan AUGE sin menoscabar la atención que se brinda al resto de los usuarios. Este punto ha sido objeto de gran polémica en la sociedad chilena, puesto que existen posiciones antagónicas entre sus actores más importantes, que van desde afirmar que las atenciones prioritarias de potenciales beneficiarios del Plan AUGE no causarán detrimentos en el acceso a atenciones médicas de pacientes con otras patologías, hasta señalar que la implementación del Plan significará crear un sistema discriminatorio(5).

Sin embargo, al implementar acciones para satisfacer determinadas exigencias, como el acceso, no hay modelo que pueda sustraerse de hacer discriminaciones que susciten reparos sobre su justicia o transparencia. La causa no es otra que la lógica limitación de recursos ante demandas sanitarias que exceden las disponibilidades(6), por lo que la determinación de prioridades en los servicios de atención de salud resulta inevitable.

Ello sucede en todos los países del mundo, independiente de que sus sistemas de atención de salud tengan un carácter principalmente público o privado, del monto de recursos con que se cuente o de la orientación de las políticas sociales.

La priorización en el Plan AUGE fue realizada sobre la base de análisis sanitarios y financieros. Las garantías son determinadas por el Ministerio de Salud, con base en estudios epidemiológicos que identifican un listado de prioridades y de intervenciones que consideran la situación de salud de la población, la efectividad de 
las intervenciones, su contribución a la extensión o a la calidad de vida y, cuando sea posible, su relación costo-efectividad. Sobre esta base se definió un listado de enfermedades y sus prestaciones asociadas.

El AUGE prioriza soluciones costo-efectivas (la mayor efectividad posible para resolver un problema de salud al menor costo), expresadas a través de protocolos los que, en teoría, deben considerar para enfrentar cada patología aquellas soluciones que tienen la mejor relación efecto-costo.

Además, garantiza derechos a la gente (para el grupo de patologías priorizadas) lo que representa un gran avance en el ámbito de los derechos ciudadanos.

Sin embargo, este proyecto incluido en la reforma de salud provoca una serie de cuestionamientos que tienen relación con la situación de incertidumbre o restricciones en que quedarían las personas que fueron excluidas en esta priorización y no tendrían acceso a exigir garantía alguna.

Es evidente que una de las debilidades que presenta el Plan AUGE es justamente la priorización por patologías, ya que esta es una forma común de discriminación en la atención de salud, dejando una sensación de injusticia o marginación por parte de quienes queden fuera de esa lista favorecida por "garantías explícitas".

Ante lo expuesto, queda de manifiesto que, frente a una sociedad que presenta desigualdades en su nivel de salud, se requiere de principios éticos que rijan la asignación de los recursos.

Como principio ético se considera fundamental el principio de justicia como lo plantea John Rawls en su libro "Teoría de la Justicia", en el que establece tres grandes principios orientados a la búsqueda de la equidad en la distribución de los valores sociales: "Todos los valores sociales -libertad, oportunidad e ingreso y riqueza-, así como las bases sociales y el respeto a sí mismo, habrán de ser distribuidas igualitariamente, a menos que una distribución desigual redunde en una ventaja para todos.

Todos los valores sociales -libertad, y oportunidad y ingreso y riqueza-, así como las bases sociales y el respeto a sí mismo, habrán de ser distribuidas igualitariamente, a menos que una distribución desigual redunde en beneficio de los menos aventajados.
Las desigualdades económicas y sociales han de ser estructuradas de manera que sean de mayor beneficio de los menos aventajados, de acuerdo a un principio de ahorro justo. Unido a que los cargos y las funciones sean accesibles a todos, bajo condiciones de justa igualdad"(7).

Esta concepción de justicia, basada en el modelo de Rawls, es aplicable a la salud en la medida en que se establezcan cuáles son las necesidades en salud que deben considerarse como bienes primarios y, por lo tanto, que deban ser garantizados para todos por igual.

La justicia se entiende así como principio ético básico en un área donde las necesidades son múltiples y se cuenta con recursos escasos para poder garantizar un nivel mínimo de salud que abarque toda la sociedad, independiente de la patología que lo afecte.

\section{Conclusión}

Ante la necesidad de una distribución equitativa de recursos que mejoren el nivel de salud de la población, se considera la salud como un bien, ya que nos permite un desarrollo integral individual y como sociedad. Por lo tanto, para el logro de equidad en salud es necesario comprender la importancia de garantizar una justa distribución de los recursos, que evite las restricciones o privilegios a los que arbitrariamente pueden estar sometidas las personas en la atención de salud.

Aun cuando los derechos económicos, sociales y culturales están condicionados en su aplicación a los recursos que el Estado disponga, son derechos exigibles en justicia y, por tanto, obligaciones perfectas y no caridad.

Un sistema de atención médica justo requiere de un diseño de normativas deontológicas que definan el marco de acción en términos de los derechos de la sociedad, las responsabilidades del Estado con los ciudadanos, las responsabilidades individuales y la forma en que se distribuirán las riquezas del Estado. Será necesario entonces que se cumpla con la dimensión teleológica que garantice una eficiente administración de los recursos disponibles, para lo cual será indispensable: a) que el sistema sanitario sea eficaz, es decir, que garantice que los métodos diagnósticos y terapéuticos sean de utilidad científica demostrada, b) que el sistema sea eficiente, esto es, que los procedimientos de diagnóstico y tratamiento sean analizados de acuerdo con criterios de costo-beneficio. 


\section{Referencias}

1. Gracia D. Profesión médica, investigación y justicia sanitaria. Bogotá: Editorial El Búho; 2002.

2. Montoya C, Barilari E, Madrid S, Ramírez M. Medición de la inequidad en la situación y atención de salud. El ejemplo de Chile, 1992. Rev Chil Salud Pública 1998; 2: 122-128.

3. Whitehead M. The concept and principles of Equity and Health. Copenhagen: OMS; 1990.

4. MINSAL. Piloto AUGE 2004. Documento para la aplicación del sistema AUGE en las redes de atención del Sistema Nacional de Servicios de Salud. Versión 1.1. Santiago de Chile: Ministerio de Salud; 2004.

5. González F. Implementación del plan AUGE en pacientes con IRC. Rev Médica de Chile 2003;131(5): 545-551.

6. Rosselot E. Aspectos Bioéticos en la Reforma de la Atención de Salud en Chile. II, Discriminación, libre elección y consentimiento informado. Rev Médica de Chile 2003;131(11): 1329-1336.

7. Rawls J. Teoría de la Justicia. México: Fondo de cultura económica; 1979.

Recibido: 3 de abril de 2008

Aceptado: 18 de mayo de 2008 